\title{
On Approximate Solutions of Functional Equations in Vector Lattices
}

\author{
Bogdan Batko ${ }^{1,2}$ \\ ${ }^{1}$ Institute of Mathematics, Pedagogical University of Cracow, Podchorą̇ych 2, 30-084 Kraków, Poland \\ ${ }^{2}$ Department of Mathematics, WSB-NLU, Zielona 27, 33-300 Nowy Sacz, Poland \\ Correspondence should be addressed to Bogdan Batko; bbatko@up.krakow.pl
}

Received 28 December 2013; Accepted 26 February 2014; Published 7 April 2014

Academic Editor: Jaeyoung Chung

Copyright (c) 2014 Bogdan Batko. This is an open access article distributed under the Creative Commons Attribution License, which permits unrestricted use, distribution, and reproduction in any medium, provided the original work is properly cited.

\begin{abstract}
We provide a method of approximation of approximate solutions of functional equations in the class of functions acting into a Riesz space (algebra). The main aim of the paper is to provide a general theorem that can act as a tool applicable to a possibly wide class of functional equations. The idea is based on the use of the Spectral Representation Theory for Riesz spaces. The main result will be applied to prove the stability of an alternative Cauchy functional equation $F(x+y)+F(x)+F(y) \neq 0 \Rightarrow F(x+y)=F(x)+F(y)$ in Riesz spaces, the Cauchy equation with squares $F(x+y)^{2}=(F(x)+F(y))^{2}$ in $f$-algebras, and the quadratic functional equation $F(x+y)+F(x-y)=2 F(x)+2 F(y)$ in Riesz spaces.
\end{abstract}

\section{Introduction}

In this paper we deal with a method of treating approximate solutions of functional equations in a class of functions taking values in Riesz spaces (algebras). Some recent results concerning stability of functional equations in ordered spaces can be found in [1-7].

In view of the fact that the idea of applying the Spectral Representation Theory (SRT for short) for Riesz spaces to investigate approximate solutions of functional equations in vector lattices has appeared fruitful for various functional equations (cf. $[2,3,5]$ ) it seems to be valuable to formulate a general theorem that could play a role of a tool hopefully applicable to a wide class of functional equations. The main purpose of this paper is to provide such a result (see Section 3).

As it has already been mentioned, in the following we are going to make use of the SRT for Riesz spaces that provides a representation of vectors of a given Riesz space $L$ by extended (admitting infinite values) real continuous functions on a certain topological space $X$ which are finite on a dense subset of $X\left(C^{\infty}(X)\right)$. The above means that a given Riesz space $L$ (under some additional assumptions) is Riesz isomorphic with a Riesz subspace of $C^{\infty}(X)$. Unfortunately, it appears that, in general, the whole of $C^{\infty}(X)$ is not necessarily a
Riesz space and that causes some difficulties. The second inconvenience we have to defeat stems from the fact that functions from $C^{\infty}(X)$ may attain infinite values.

Once the main results of the paper are achieved, we show their benefits. We apply them to investigate approximate solutions of three selected functional equations. The first two of them have the common origin, but they exhibit different stability behaviours (at least in the class of real-valued functions). We show that an alternative Cauchy functional equation

$$
F(x+y)+F(x)+F(y) \neq 0 \Longrightarrow F(x+y)=F(x)+F(y)
$$

is stable in Riesz spaces (see Section 4). In Section 5 we prove that the Cauchy equation with squares

$$
F(x+y)^{2}=(F(x)+F(y))^{2}
$$

is stable in $f$-algebras; however unlike in the case of realvalued functions it is not superstable. The third one is the quadratic functional equation

$$
F(x+y)+F(x-y)=2 F(x)+2 F(y) .
$$

We prove its stability in Riesz spaces in Section 6. 


\section{Preliminaries}

Throughout the paper $\mathbb{N}, \mathbb{Z}, \mathbb{R}$, and $\mathbb{R}_{+}$are used to denote the sets of all positive integers, integers, real numbers, and nonnegative real numbers, respectively.

For the reader's convenience we quote basic definitions and properties concerning Riesz spaces following [8].

We say that a partially ordered real linear space $L$ (we denote the order in $L$ by $\leq$ ) is a Riesz space (vector lattice) if $\sup \{x, y\}$ exists for all $x, y \in L$ (cf. [8, Definition 11.1]). We define the absolute value of $x \in L$ by the formula $|x|:=$ $\sup \{x,-x\} \geq 0$. A Riesz space $L$ is called Archimedean if, for each $x \in L$, the inequality $x \leq 0$ holds whenever the set $\{n x: n \in \mathbb{N}\}$ is bounded from above (cf. [8, Definition 22.1]). We say that $L$ is a Riesz algebra if $L$ is a Riesz space equipped with the common algebra multiplication satisfying $x y \geq 0$ whenever $x, y \geq 0$. A Riesz algebra $L$ is termed an $f$-algebra, whenever $\inf \{x, y\}=0 \operatorname{implies} \inf \{x z, y\}=\inf \{z x, y\}=0$ for every $z \geq 0$.

A Riesz space $L$ is said to be Dedekind complete ( $\sigma$ complete) if any nonempty (at most countable) subset of $L$ which is bounded from above has a supremum (cf. [8, Definition 1.1]).

In the following the notion of the relatively uniform convergence will be used (cf. [8, Definition 39.1]). Let $L$ be a Riesz space and let $u \in L_{+}:=\{u \in L: u \geq 0\}$. A sequence $\left(f_{n}\right)_{n \in \mathbb{N}}$ in $L$ is said to converge $u$-uniformly to an element $f \in L$ whenever, for every $\varepsilon>0$, there exists a positive integer $n_{0}$ such that $\left|f-f_{n}\right| \leq \varepsilon u$ holds for all $n \geq n_{0}$. We say that $\left(f_{n}\right)_{n \in \mathbb{N}}$ is relatively uniformly convergent if $\left(f_{n}\right)_{n \in \mathbb{N}}$ is $u$ uniformly convergent with some $u \in L_{+}$. A sequence $\left(f_{n}\right)_{n \in \mathbb{N}}$ in $L$ is called $u$-uniform Cauchy sequence whenever, for every $\varepsilon>0$, there exists a positive integer $n_{1}$ such that $\left|f_{m}-f_{n}\right| \leq \varepsilon u$ holds for all $m, n \geq n_{1}$.

In general the $u$-uniform limit of a sequence may depend on the choice of $u \in L_{+}$and does not have to be unique. However, if $L$ is Archimedean, the $u$-uniform limit, if it exists, is unique. In this case the fact that $\left(f_{n}\right)_{n \in \mathbb{N}}$ converges $u$ uniformly to $f$ will be denoted by $\lim _{n \rightarrow \infty}^{u} f_{n}=f$.

A Riesz space $L$ is called $u$-uniformly complete (with a given $u \in L_{+}$) whenever every $u$-uniform Cauchy sequence has a $u$-uniform limit. We say that $L$ is uniformly complete if it is $u$-uniformly complete with every $u \in L_{+}$(cf. [8, Definition 39.3]).

There is a large class of spaces satisfying the above conditions. In particular every Dedekind $\sigma$-complete space is Archimedean and uniformly complete.

The element $e \in L_{+}$is called a strong unit if for every $l \in L$ there exists $\alpha \in \mathbb{R}$ such that $|l| \leq \alpha e$.

The element $e \in L_{+}$is called a weak unit if the band generated by $e$ is the whole of $L$ (cf. [8, Definition 21.4]). Recall that a Riesz subspace $M$ of $L$ is an ideal if it is solid, that is, whenever it follows from $f \in M, g \in L$, and $|g| \leq|f|$ that $g \in M$. An ideal $M$ is termed a band in $L$, whenever a subset of $M$ has a supremum in $L$, that supremum is an element of $M$ (cf. [8, Definition 17.1]). If $L$ is Archimedean then $e \in L_{+}$ is a weak unit if and only if $\{e\}^{\perp}=\{0\}$, where $\{e\}^{\perp}$ stands for the disjoint complement of $e$ (cf. $[9,353 \mathrm{~L}])$.
A linear mapping $\pi: L \rightarrow M$ between Riesz spaces $L$ and $M$ is called a Riesz homomorphism if

$$
\pi(\sup \{v, w\})=\sup \{\pi v, \pi w\} \quad \text { for } v, w \in L .
$$

Now we define the family $C^{\infty}(X)$ of extended (admitting infinite values) real continuous functions on a given topological space $X$ that are finite-valued on a dense subset of $X$ and discuss their elementary properties.

Given a topological space $X$, any continuous mapping $f$ of $X$ into $R^{\infty}:=\mathbb{R} \cup\{-\infty\} \cup\{+\infty\}$ with the usual topology, such that the set

$$
R(f)=\{x:|f(x)|<\infty\}
$$

is dense in $X$, is called an extended (real-valued) continuous function on $X$. The set of all extended (real-valued) continuous functions on $X$ will be denoted by $C^{\infty}(X)$. We consider the pointwise order in $C^{\infty}(X)$ and the pointwise multiplication by scalars, where it is understood that $0 \cdot \infty=$ 0 . For any $f \in C^{\infty}(X)$, the set $R(f)$ is open and dense. If $f, g, h \in C^{\infty}(X)$ and $h(x)=f(x)+g(x)$ holds for all $x \in R(f) \cap R(g)$, then (by definition) $h$ is called the sum of $f$ and $g$ (notation $h=f+g$ ). Since the set $R(f) \cap R(g)$ is dense, the function $h=f+g$ is uniquely defined if it exists. It occurs that $C^{\infty}(X)$ is not necessarily closed with respect to the operation of addition (cf. [8, p. 295]). Any subset of $C^{\infty}(X)$ closed under the operation of addition, multiplication by scalars and the taking of finite infima and suprema, is obviously a Riesz space with respect to pointwise ordering. Accordingly, any subset of this kind is called a Riesz space of extended real continuous functions on $X$.

The difficulties with the addition disappear if $X$ is extremally disconnected; that is, the closure of every open set is open. Then $C^{\infty}(X)$ is a Riesz space (cf. [8, Theorem $47.2]$ ) and even an $f$-algebra with the multiplicative identity under the appropriate definition of the multiplication. Given $f, g \in C^{\infty}(X)$, the set $R(f) \cap R(g)$ is open and dense. Thus the function which is equal to $f(x) g(x)$ for every $x \in R(f) \cap R(g)$ is finite-valued and continuous on $R(f) \cap R(g)$. Hence, this function can uniquely be extended to an extended continuous function on $X$ (cf. [8, Theorem 47.1]), and let, by definition, this extended function be $f g$.

Proposition 1 (cf. [3, Proposition 1]). If $X$ is an extremally disconnected topological space then $C^{\infty}(X)$ is an $f$-algebra with a multiplicative identity.

At the end of this section we briefly remind the notion of the Hyers-Ulam stability originated by the well-known problem posed by Ulam (cf. [10]) during his talk at the University of Wisconsin in 1940 and the answer given by Hyers (cf. [11]), which we quote below.

Let $X$ and $Y$ be Banach spaces and let $\varepsilon>0$. Then for every $g: X \rightarrow Y$ with $\sup _{x, y \in X}\|g(x+y)-g(x)-g(y)\| \leq \varepsilon$ there is a unique $f: X \rightarrow Y$ such that $\sup _{x \in X}\|g(x)-f(x)\| \leq \varepsilon$ and

$$
f(x+y)=f(x)+f(y) \quad \text { for } x, y \in X
$$


To describe this result we used to say that the Cauchy functional equation (6) is Hyers-Ulam stable in the class of functions $Y^{X}$. It is worth to mention here that, probably, the first known result in this direction is due to Pólya and Szegö (cf. [12]).

Next, the stability of functional equations has been widely investigated and generalized in various directions by many authors. For the extensive discussion concerning possible definitions of the stability of functional equations and differences between them we refer the interested reader to [13]. Examples of various recent results concerning the subject as well as a list of numerous references connected with it can be found in the survey paper [14].

\section{Main Results}

From now on let $(G,+)$ be a groupoid and $L$ a Riesz space $(f$ algebra) and let $\mathscr{E}: L^{G} \rightarrow L^{G^{k}} ; k \in \mathbb{N}$. We will say that a function $F: G \rightarrow L$ is a solution of equation

$$
\mathscr{E} F=0
$$

if $\mathscr{E} F(\bar{x})=0$ for $\bar{x} \in G^{k}$. Given $u \in L_{+}$, any $F: G \rightarrow L$ with

$$
|\mathscr{E} F(\bar{x})| \leq u \quad \text { for } \bar{x} \in G^{k}
$$

will be called a $u$-solution of (7). $F$ will be termed an approximate solution of (7) if it is a $u$-solution of (7) with some $u \in L_{+}$. Finally, we will say that (7) is stable (or Hyers-Ulam stable) if for any $u \in L_{+}$there is $\Delta(u) \in L_{+}$such that for each $u$-solution $F: G \rightarrow L$ of $(7)$ there exists a solution $A: G \rightarrow L$ of (7) with

$$
|F(x)-A(x)| \leq \Delta(u) \quad \text { for } x \in G .
$$

We will focus on a class of functional equations that possess the following property.

Definition 2. We will say that (7) has the uniform $\mathbb{R}$ approximation property (URAP for short) if there exist $\mathscr{H}_{n}$ : $L^{G} \rightarrow L^{G},(n \in \mathbb{N}), \delta: \mathbb{R} \rightarrow \mathbb{R}$, and real sequences $\alpha_{n} \rightarrow 0$, $\beta_{n} \rightarrow 0, \gamma_{n} \rightarrow \gamma \in \mathbb{R}$, and $\rho_{n} \rightarrow 0$ such that if we take $\mathbb{R}$, with the ordinary order, as a realisation of $L$ then for any $\varepsilon>0$ and any $\varepsilon$-solution $f: G \rightarrow \mathbb{R}$ of (7) the following conditions hold:

(P1) $\left|\mathscr{H}_{n} f(x)-\mathscr{H}_{m} f(x)\right| \leq\left(\alpha_{n}+\beta_{m}\right) \delta(\varepsilon)$,

(P2) $\left|\mathscr{H}_{n} f(x)-f(x)\right| \leq \gamma_{n} \delta(\varepsilon)$,

(P3) $\left|\mathscr{E} \mathscr{H}_{n} f(\bar{x})\right| \leq \rho_{n} \delta(\varepsilon)$,

for $x \in G, \bar{x} \in G^{k}$, and $m, n \in \mathbb{N}$.

The URAP is closely related to the Hyers-Ulam stability of (7) in the class of real-valued functions, where the role of the operators $\mathscr{H}_{n},(n \in \mathbb{N})$, is played by the so called Hyers operators (cf. $[10,11])$. The term uniform in the name of the property refers to the fact that the right-hand sides of (P1)(P3) do not depend on $f$. It is evident that URAP implies the Hyers-Ulam stability. As it will be shown below, in many cases the converse is also true.
Lemma 3. Let $(G,+)$ be a groupoid. Assume that $(7)$ is HyersUlam stable in the class of real-valued functions defined on $G$ and that there exist $\mu_{n}, e_{n} \rightarrow \infty$ and $\eta_{n}: G \rightarrow G,(n \in \mathbb{N})$, such that for any solution $a: G \rightarrow \mathbb{R}$ of (7)

$$
a \circ \eta_{n}=\mu_{n} a \quad \text { for } n \in \mathbb{N}
$$

and for any $\mathcal{E}$-solution $f: G \rightarrow \mathbb{R}$ of $(7)$

$$
\frac{1}{e_{n}} \mathscr{E} f=\mathscr{E} \frac{1}{\mu_{n}} f \quad \text { for } n \in \mathbb{N} .
$$

Then (7) possesses the URAP.

Proof. Let $f: G \rightarrow \mathbb{R}$ be an $\varepsilon$-solution of (7). We define $\mathscr{H}_{n} f(x):=f\left(\eta_{n}(x)\right) / \mu_{n}$ for $x \in G, n \in \mathbb{N}$. By the Hyers-Ulam stability of (7) there exist a solution $a: G \rightarrow \mathbb{R}$ of (7) and $\delta(\varepsilon) \in \mathbb{R}$ with

$$
|f(x)-a(x)| \leq \delta(\varepsilon) \quad \text { for } x \in G
$$

Applying (12) for $\eta_{n}(x)$ in place of $x$ and taking into account (10) we obtain

$$
\left|f\left(\eta_{n}(x)\right)-\mu_{n} a(x)\right| \leq \delta(\varepsilon) \quad \text { for } x \in G, n \in \mathbb{N} .
$$

Dividing the above inequality by $\mu_{n}$, side by side, we have

$$
\left|\mathscr{H}_{n} f(x)-a(x)\right| \leq \frac{1}{\mu_{n}} \delta(\varepsilon) \quad \text { for } x \in G, n \in \mathbb{N}
$$

on account of the definition of $\mathscr{H}_{n}$. By (14) and (12) we have (P2) with $\gamma_{n}:=1+\left(1 / \mu_{n}\right)$.

If we rewrite (14) for given $n \in \mathbb{N}$ and $m \in \mathbb{N}$ and then add the resulting inequalities, side by side, we obtain (P1) with $\alpha_{n}=1 / \mu_{n}$ and $\beta_{m}=1 / \mu_{m}$.

Since $f$ is an $\varepsilon$-solution of (7) then using (8) for $\eta_{n}(\bar{x}):=$ $\left(\eta_{n}\left(x_{1}\right), \ldots, \eta_{n}\left(x_{k}\right)\right)$ in place of $\bar{x}$ we have

$$
\left|\mathscr{E} f\left(\eta_{n}(\bar{x})\right)\right| \leq \varepsilon \quad \text { for } \bar{x} \in G^{k}, n \in \mathbb{N} .
$$

Dividing the above inequality by $e_{n}$ and taking into account (11), in view of the definition of $\mathscr{H}_{n}$, we arrive at

$$
\left|\mathscr{E} \mathscr{H}_{n} f(\bar{x})\right| \leq \frac{1}{e_{n}} \varepsilon \text { for } \bar{x} \in G^{k}, n \in \mathbb{N},
$$

which means that (P3) holds with $\rho_{n}=\varepsilon / e_{n} \delta(\varepsilon)$ provided that $\delta(\varepsilon) \neq 0$. The case $\delta(\varepsilon)=0$ means that $f$ satisfies (7) and, therefore, (P3) holds with any nonnegative $\rho_{n}$.

Remark 4. Let us observe that all the assumptions of Lemma 3 are fulfilled if we assume that (7) is Hyers-Ulam stable in the class of real-valued functions and that there exists $\lambda_{n} \rightarrow \infty$ such that any solution $a: G \rightarrow \mathbb{R}$ of $(7)$ is $\left(\lambda_{n}, \alpha\right)$ homogeneous with some $\alpha>0$; that is,

$$
a\left(\lambda_{n} x\right)=\lambda_{n}^{\alpha} a(x) \quad \text { for } x \in G, n \in \mathbb{N},
$$

and $\mathscr{E}$ is $\left(\lambda_{n}^{-\alpha}, \beta\right)$-homogeneous with some $\beta>0$; that is,

$$
\mathscr{E}\left(\lambda_{n}^{-\alpha} f\right)=\lambda_{n}^{-\alpha \beta} \mathscr{E} f \quad \text { for } n \in \mathbb{N}, f \in \mathbb{R}^{G} .
$$


In particular, any functional equation which is HyersUlam stable in the class of real-valued functions, whose solution form additive functions, has the URAP.

Corollary 5. Let $f: G \rightarrow \mathbb{R}$ map a groupoid $(G,+)$ into $\mathbb{R}$. Assume that there exist an additive function $a: G \rightarrow \mathbb{R}$ and a real number $\alpha \geq 0$ such that

$$
|f(x)-a(x)| \leq \alpha \quad \text { for } x \in G .
$$

Let $\mathscr{H}_{n} f(x):=(1 / n) f(n x)$ for $x \in G$ and $n \in \mathbb{N}$. Then $(P 1)-$ (P3) hold with $\alpha_{n}=\beta_{n}=1 / n, \gamma_{n}=1+1 / n, \rho_{n}=3 / n$, for $n \in \mathbb{N}$ and $\delta=\alpha$.

Proof. Routine.

Let $\left\langle x_{1}, x_{2}, \ldots, x_{k}\right\rangle$ stand for the substructure of $G$ generated by $x_{1}, \ldots, x_{k} \in G$ and let $\Delta \in L$. We will consider the following hypotheses:

(H1) there exist a topological space $X$ and a Riesz ( $f$ algebra) isomorphism $\pi: L \rightarrow \widehat{L} \subset C^{\infty}(X)$,

(H2) $\pi$ and $\mathscr{E}$ commute; that is, $\pi(\mathscr{E} F(\bar{x}))=\mathscr{E}(\pi F)(\bar{x})$ for any $F \in L^{G}$ and $\bar{x} \in G^{k}$,

(H3) $\pi$ and $\mathscr{H}_{n}$ commute; that is, $\pi\left(\mathscr{H}_{n} F(x)\right)=\left(\mathscr{H}_{n} \pi F\right)(x)$ for any $F \in L^{G}, x \in G, n \in \mathbb{N}$,

(H4) for each $\bar{x}=\left(x_{1}, x_{2}, \ldots x_{k}\right) \in G^{k}$ there exists an open and dense subset $w_{\bar{x}}$ of $X$ such that

$$
w_{\bar{x}} \subset R(\pi F(z)) \quad \text { for } z \in\left\langle x_{1}, x_{2}, \ldots, x_{k}\right\rangle
$$

(H5) for each $\bar{x} \in G^{k}$, if $\lim _{n \rightarrow \infty} \Delta^{\Delta} \mathscr{H}_{n} F(\bar{x})$ exists, then

$$
\lim _{n \rightarrow \infty}{ }^{\Delta} \mathscr{E} \mathscr{H}_{n} F(\bar{x})=\mathscr{E}\left(\lim _{n \rightarrow \infty}{ }^{\Delta} \mathscr{H}_{n} F\right)(\bar{x}) .
$$

Let us note that the SRT for Riesz spaces provides results which guarantee (H1). Various classical spectral representation theorems offer effective constructions of a topological space $X$ as well as a space of representatives $\widehat{L}$ and a Riesz isomorphism $\pi$, depending on the properties of a given Riesz space $L$ (cf., e.g., [8, Ch.7]).

It is easy to see that if $\mathscr{E}$ and $\mathscr{H}_{n}$ are defined with the use of the ordinary Riesz space (algebra) operations, that is, linear operations or lattice operations, then (H2), (H3), and (H5) are automatically satisfied.

Now we are going to prove a lemma that provides some properties of a function $F: G \rightarrow L$ that yield ( $\mathrm{H} 4$ ) (for $k=$ $2)$. Assume that (H1) holds and that we are given mappings $B: L \rightarrow L, C: L \times L \rightarrow L, D: L \times L \times L \rightarrow L$ and open and dense subsets $R_{C}, R_{D}$ of $X$ such that

$$
\begin{aligned}
& R(\pi B(v)) \subset R(\pi v) \\
& R(\pi v) \cap R(\pi w) \cap R_{C} \subset R(\pi C(v, w)) \\
& R(\pi v) \cap R(\pi w) \cap R(\pi t) \cap R_{D} \subset R(\pi D(v, w, t)) \\
& \quad \text { for } v, w, t \in L .
\end{aligned}
$$

For fixed $x \in G$ we consider the following hypotheses.
(L1) $(G,+)$ is an Abelian group and

$$
B(F(y)) \leq C(F(y+z), F(z)) \text { for } y, z \in\langle x\rangle,
$$

(L2) $(G,+)$ is an Abelian semigroup and

$$
B(F(y+z)) \leq C(F(y), F(z)) \text { for } y, z \in\langle x\rangle
$$

(L3) $(G,+)$ is an Abelian group and

$B(F(y+z)) \leq D(F(y), F(z), F(y-z))$ for $y, z \in\langle x\rangle$.

Lemma 6. Let $F: G \rightarrow L$ map a groupoid $(G,+)$ into a Riesz space $L$ and let (H1) hold. If for any $x \in G$ at least one of the hypotheses (L1), (L2), and (L3) holds then (H4) (for $k=2)$ is fulfilled.

Proof. Assume, at first, that (L1) holds. For fixed $x \in G$ we define

$$
w_{x}:=R(\pi F(x)) \cap R(\pi F(0)) \cap R_{C} .
$$

By (23) and (H1), for every $y, z \in\langle x\rangle$, we have

$$
\pi B(F(y)) \leq \pi C(F(y+z), F(z)),
$$

which means that

$$
R(\pi C(F(y+z), F(z))) \subset R(\pi B(F(y))) .
$$

Therefore, by (22), we see that

$$
\begin{array}{r}
R(\pi F(y+z)) \cap R(\pi F(z)) \cap R_{C} \subset R(\pi F(y)) \\
\text { for } y, z \in\langle x\rangle .
\end{array}
$$

Replacing $y$ by $-x$ and $z$ by $x$ in (29) we observe that $w_{x} \subset$ $R(\pi F(-x))$. Now, suppose that $w_{x} \subset R(\pi F(n x))$ for given $n \in \mathbb{N}$ and apply (29) with $y$ and $z$ replaced by $(n+1) x$ and $-x$, respectively, in order to obtain $w_{x} \subset R(\pi F((n+1) x))$. By induction we arrive at

$$
w_{x} \subset R(\pi F(n x)) \quad \text { for } n \in \mathbb{N} \text {. }
$$

On the other hand, using (29) with $-n x$ in place of $y$ and $n x$ in place of $z$, we receive $w_{x} \cap R(\pi F(n x)) \subset R(\pi F(-n x))$. This along with (30) yields

$$
w_{x} \subset R(\pi F(z)) \quad \text { for } z \in\langle x\rangle .
$$

For arbitrary $x_{1}, x_{2} \in G$ we define $w_{x_{1}, x_{2}}:=w_{x_{1}} \cap w_{x_{2}}$, where $w_{x_{1}}$ and $w_{x_{2}}$ are given by (26). By (31) $w_{x_{1}} \subset R(\pi F(y))$ for $y \in\left\langle x_{1}\right\rangle$ and $w_{x_{2}} \subset R(\pi F(y))$ for $y \in\left\langle x_{2}\right\rangle$. Let us consider $y \in\left\langle x_{1}\right\rangle$ and $z \in\left\langle x_{2}\right\rangle$. Using (29) with $y$ and $z$ replaced by $y+z$ and $-z$, respectively, we observe that $w_{x_{1}, x_{2}} \subset R(\pi F(y+$ $z)$ ), which completes the proof of (20).

Now we assume that (L2) holds. Let $x \in G$ and let

$$
w_{x}:=R(\pi F(x)) \cap R_{C} .
$$


Similarly as in case (L1) one can observe that (H1), (24), and (22) yield

$$
\begin{array}{r}
R(\pi F(y)) \cap R(\pi F(z)) \cap R_{C} \subset R(\pi F(y+z)) \\
\text { for } y, z \in\langle x\rangle .
\end{array}
$$

By the definition $w_{x} \subset R(\pi F(x))$. Suppose that $w_{x} \subset$ $R(\pi F(n x))$ for given $n \in \mathbb{N}$ and apply (33) with $y$ and $z$ replaced by $n x$ and $x$, respectively, in order to obtain $w_{x} \subset$ $R(\pi F((n+1) x))$. By induction we receive

$$
w_{x} \subset R(\pi F(n x)) \quad \text { for } n \in \mathbb{N} \text {; }
$$

thus (31) holds.

Fix arbitrary $x_{1}, x_{2} \in G$ and define $w_{x_{1}, x_{2}}:=w_{x_{1}} \cap w_{x_{2}}$, where $w_{x_{1}}$ and $w_{x_{2}}$ are given by (32). By (31) $w_{x_{1}} \subset R(\pi F(y))$ for $y \in\left\langle x_{1}\right\rangle$ and $w_{x_{2}} \subset R(\pi F(y))$ for $y \in\left\langle x_{2}\right\rangle$. Let us consider $y \in\left\langle x_{1}\right\rangle, z \in\left\langle x_{2}\right\rangle$. Using (33) for $y$ and $z$, we observe that $w_{x_{1}, x_{2}} \subset R(\pi F(y+z))$. This completes the proof of (20).

Finally, assume that (L3) holds. Let $x \in G$ and let

$$
w_{x}:=R(\pi F(x)) \cap R(\pi F(-x)) \cap R(\pi F(0)) \cap R_{D} .
$$

Observe that due to (H1), (25), and (22) we have

$$
\begin{gathered}
R(\pi F(y)) \cap R(\pi F(z)) \cap R(\pi F(y-z)) \cap R_{D} \\
\subset R(\pi F(y+z)) \quad \text { for } y, z \in\langle x\rangle .
\end{gathered}
$$

By the definition $w_{x} \subset R(\pi F(x)) \cap R(\pi F(0))$. Let $n \in \mathbb{N}$, suppose that $w_{x} \subset R(\pi F(k x))$ for $k \in\{0, \ldots, n\}$, and apply (36) with $y$ and $z$ replaced by $n x$ and $x$, respectively, in order to obtain $w_{x} \subset R(\pi F((n+1) x))$. By induction we obtain

$$
w_{x} \subset R(\pi F(n x)) \quad \text { for } n \in \mathbb{N} \text {. }
$$

Exchanging $x$ with $-x$ and repeating the lines of the proof of (37) we conclude that $w_{x} \subset R(\pi F(-n x))$ for $n \in \mathbb{N}$, which together with (37) results with (31).

Fix arbitrary $x_{1}, x_{2} \in G$ and define $w_{x_{1}, x_{2}}:=w_{x_{1}} \cap w_{x_{2}} \cap$ $R\left(\pi F\left(x_{1}-x_{2}\right)\right)$, where $w_{x_{1}}$ and $w_{x_{2}}$ are given by (35). By (31) $w_{x_{1}, x_{2}} \subset R(\pi F(y))$ for $y \in\left\langle x_{1}\right\rangle$ and $w_{x_{1}, x_{2}} \subset R(\pi F(y))$ for $y \in\left\langle x_{2}\right\rangle$. We will prove that $w_{x_{1}, x_{2}} \subset R\left(\pi F\left(k x_{1}+l x_{2}\right)\right)$ for $k, l \in \mathbb{Z}$. It is evident that $w_{x_{1}, x_{2}} \subset R\left(\pi F\left(x_{1}+x_{2}\right)\right)$. Let $k \in \mathbb{N}$ and suppose that $w_{x_{1}, x_{2}} \subset R\left(\pi F\left(m x_{1}+x_{2}\right)\right)$ for $m \in\{0, \ldots, k\}$. Applying (36) with $y$ and $z$ replaced by $m x_{1}+x_{2}$ and $x_{1}$, respectively, we observe that $w_{x_{1}, x_{2}} \subset R\left(\pi F\left((m+1) x_{1}+x_{2}\right)\right)$. Thus, by induction

$$
w_{x_{1}, x_{2}} \subset R\left(\pi F\left(k x_{1}+x_{2}\right)\right) \text { for } k \in \mathbb{N} .
$$

To observe that (38) holds also for negative integers it is enough to apply (36) with $x_{2}$ and $-k x_{1}$ in place of $y$ and $z$, respectively, and take into account (38). Thus,

$$
w_{x_{1}, x_{2}} \subset R\left(\pi F\left(k x_{1}+x_{2}\right)\right) \text { for } k \in \mathbb{Z} .
$$

Now, let $k \in \mathbb{Z}$ be fixed and assume that, for given $l \in \mathbb{N}$, it is $w_{x_{1}, x_{2}} \subset R\left(\pi F\left(k x_{1}+m x_{2}\right)\right)$ for $m \in\{0, \ldots, l\}$. Then by (36) with $y$ and $z$ replaced by $k x_{1}+l x_{2}$ and $x_{2}$, respectively, we obtain $w_{x_{1}, x_{2}} \subset R\left(\pi F\left(k x_{1}+(l+1) x_{2}\right)\right)$. By induction,

$$
w_{x_{1}, x_{2}} \subset R\left(\pi F\left(k x_{1}+l x_{2}\right)\right) \quad \text { for } k \in \mathbb{Z}, l \in \mathbb{N} \text {. }
$$

If $l$ is a negative integer, we use (36) with $y$ and $z$ replaced by $k x_{1}$ and $l x_{2}$, respectively, together with (40) to complete the proof of (20).

Now we are in a position to formulate and prove the main theorem of the paper.

Theorem 7. Let $(G,+)$ be a groupoid, let $L$ be an Archimedean Riesz space, and let (7) possess the URAP. Assume that, for given $u \in L_{+}, F: G \rightarrow L$ is a $u$-solution of (7) and that hypotheses (H1)-(H5) hold. Let $\Delta(u) \in L$ be such that $\pi \Delta(u)(s) \geq \delta(\pi u(s))$ for $s \in R(\pi \Delta(u))$ and assume that $L$ is $\Delta(u)$-uniformly complete. Then there exists a solution $A: G \rightarrow$ L of (7) such that

$$
|F(x)-A(x)| \leq \gamma \Delta(u) \quad \text { for } x \in G .
$$

Moreover, the solution of (7) satisfying (41) is unique provided that it is unique in the case where we consider $\mathbb{R}$ as a realisation of $L$.

Proof. The proof runs in three steps.

Step 1. Assume that $F: G \rightarrow L$ is a $u$-solution of (7). We will prove that, for every $x \in G$, the sequence $\left(\mathscr{H}_{n} F(x)\right)_{n \in \mathbb{N}}$ is $\Delta(u)$-uniformly convergent.

Fix $x \in G$. By (H4) there exists an open and dense subset $w_{x}$ of $X$ with

$$
w_{x} \subset R(\pi F(z)) \quad \text { for } z \in\langle x\rangle \text {. }
$$

Let $W_{x}:=w_{x} \cap R(\pi u) \cap R(\pi \Delta(u))$. By (H1) and (H2) for arbitrarily fixed $s \in W_{x}$ and $\bar{x} \in\langle x\rangle^{k}$ we have

$$
|\mathscr{E}(\pi F(\cdot)(s))(\bar{x})| \leq \pi u(s)
$$

where $\pi F(\cdot)(s)$ stands for the function mapping $G$ into $\mathbb{R}^{\infty}$ given by $\pi F(x)(s):=\pi(F(x))(s)$ for $x \in G$. This means that $\left.\pi F(\cdot)(s)\right|_{\langle x\rangle}$ is a real-valued $\pi u(s)$-solution of (7) on $\langle x\rangle$. Since (7) has the URAP, by (P1),

$$
\left|\mathscr{H}_{n} \pi F(\cdot)(s)(x)-\mathscr{H}_{m} \pi F(\cdot)(s)(x)\right| \leq\left(\alpha_{n}+\beta_{m}\right) \delta(\pi u(s)),
$$

with some $\alpha_{n} \rightarrow 0$ and $\beta_{m} \rightarrow 0$. Similarly, by (P2) we obtain

$$
\left|\mathscr{H}_{n} \pi F(\cdot)(s)(x)-\pi F(x)(s)\right| \leq \gamma_{n} \delta(\pi u(s)), \quad \text { for } n \in \mathbb{N}
$$

with some $\gamma_{n} \rightarrow \gamma$. According to (H3) and the definition of $\Delta$ the above inequalities imply

$$
\begin{aligned}
& \left|\pi \mathscr{H}_{n} F(x)(s)-\pi \mathscr{H}_{m} F(x)(s)\right| \\
& \quad \leq\left(\alpha_{n}+\beta_{m}\right) \pi \Delta(u)(s), \quad \text { for } n, m \in \mathbb{N}, \\
& \left|\pi \mathscr{H}_{n} F(x)(s)-\pi F(x)(s)\right| \\
& \quad \leq \gamma_{n} \pi \Delta(u)(s), \quad \text { for } n \in \mathbb{N},
\end{aligned}
$$


respectively. Since $s \in W_{x}$ is arbitrary, $W_{x}$ is open and dense in $X$; moreover all the functions in the above inequalities (as functions of variable $s$ ) are continuous; we obtain

$$
\begin{array}{r}
\left|\pi \mathscr{H}_{n} F(x)-\pi \mathscr{H}_{m} F(x)\right| \leq\left(\alpha_{n}+\beta_{m}\right) \pi \Delta(u), \\
\text { for } n, m \in \mathbb{N}, \\
\left|\pi \mathscr{H}_{n} F(x)-\pi F(x)\right| \leq \gamma_{n} \pi \Delta(u), \quad \text { for } n \in \mathbb{N} .
\end{array}
$$

Due to the fact that $\pi$ is a Riesz homomorphism, the above inequalities result in

$$
\begin{gathered}
\left|\mathscr{H}_{n} F(x)-\mathscr{H}_{m} F(x)\right| \leq\left(\alpha_{n}+\beta_{m}\right) \Delta(u), \quad \text { for } n, m \in \mathbb{N} \\
\left|\mathscr{H}_{n} F(x)-F(x)\right| \leq \gamma_{n} \Delta(u), \quad \text { for } n \in \mathbb{N},
\end{gathered}
$$

respectively. Inequality (48) means that $\left(\mathscr{H}_{n} F(x)\right)_{n \in \mathbb{N}}$ is a $\Delta(u)$-uniform Cauchy sequence in a $\Delta(u)$-uniformly complete Riesz space and, therefore, relatively uniformly convergent. This, due to the fact that $x \in G$ was arbitrarily fixed, proves that $A: G \rightarrow L$ given by

$$
A(x):=\lim _{n \rightarrow \infty}{ }^{\Delta(u)} \mathscr{H}_{n} F(x), \quad \text { for } x \in G
$$

is well defined.

Letting $n \rightarrow \infty$ in (49) we obtain (41) as $L$ is Archimedean.

Step 2. We will prove that $A$ is a solution of (7). Let $\bar{x}=$ $\left(x_{1}, x_{2}, \ldots, x_{k}\right) \in G^{k}$. By (H4) there exists an open and dense subset $w_{\bar{x}}$ of $X$ with (20). Let $W_{\bar{x}}:=w_{\bar{x}} \cap R(\pi u) \cap R(\pi \Delta(u))$. By (H1) and (H2) for arbitrarily fixed $s \in W_{x}$ and $\bar{y} \in$ $\left\langle x_{1}, x_{2}, \ldots, x_{k}\right\rangle^{k}$ we have

$$
|\mathscr{E} \pi F(\cdot)(s)(\bar{y})| \leq \pi u(s)
$$

which means that $\left.\pi F(\cdot)(s)\right|_{\left\langle x_{1}, x_{2}, \ldots, x_{k}\right\rangle}$ is a real-valued $\pi u(s)$ solution of (7) on $\left\langle x_{1}, x_{2}, \ldots, x_{k}\right\rangle$. Since (7) has the URAP, by (P3),

$$
\left|\mathscr{E} \mathscr{H}_{n} \pi F(\cdot)(s)(\bar{x})\right| \leq \rho_{n} \delta(\pi u(s)) \quad \text { for } n \in \mathbb{N}
$$

with some $\rho_{n} \rightarrow 0$ and consequently, taking into account the definition of $\Delta,(\mathrm{H} 2)$, and (H3), we have

$$
\left|\pi \mathscr{E} \mathscr{H}_{n} F(\bar{x})(s)\right| \leq \rho_{n} \pi \Delta(u)(s) \quad \text { for } n \in \mathbb{N} \text {. }
$$

Since the last inequality is valid for any $s$ from the open and dense subset $W_{\bar{x}}$ of $X$ and all the functions in the above inequality are continuous, we obtain

$$
\left|\pi \mathscr{C} \mathscr{H}_{n} F(\bar{x})\right| \leq \rho_{n} \pi \Delta(u) \quad \text { for } n \in \mathbb{N} .
$$

Finally, taking into account the fact that $\pi$ is a Riesz homomorphism, we arrive at

$$
\left|\mathscr{E} \mathscr{H}_{n} F(\bar{x})\right| \leq \rho_{n} \Delta(u) \quad \text { for } n \in \mathbb{N} \text {. }
$$

Letting $n \rightarrow \infty$ and taking into account the definition of $A$, (H5) and the fact that $L$ is Archimedean, we have $\mathscr{E} A(\bar{x})=0$ which proves that $A$ satisfies (7) as $\bar{x} \in G^{k}$ was chosen arbitrarily.

Step 3. We will prove the uniqueness of $A$ satisfying (41) under the assumption that in the class of real-valued functions a solution of (7) which approximates $F$ is uniquely determined. Contrary, suppose that two solutions $A_{1}, A_{2}: G \rightarrow L$ of (7) satisfy

$$
\left|F(x)-A_{i}(x)\right| \leq \gamma \Delta(u) \quad \text { for } x \in G, i \in\{1,2\} .
$$

Fix $x \in G$. By (H4) there exists an open and dense subset $w_{x}$ of $X$ such that

$$
w_{x} \subset R(\pi F(z)) \quad \text { for } z \in\langle x\rangle \text {. }
$$

Let $W_{x}:=w_{x} \cap R(\pi u) \cap R(\pi \Delta(u))$. According to $(\mathrm{H} 1)$ and $(\mathrm{H} 2)$, the above means that, for arbitrarily fixed $s \in W_{x}$, function $\left.\pi F(\cdot)(s)\right|_{\langle x\rangle}$ is a real-valued $\pi u(s)$-solution of (7) on $\langle x\rangle$. On the other hand, by (56),

$$
\left|\pi F(y)(s)-\pi A_{i}(y)(s)\right| \leq \gamma \pi \Delta(u)(s) \text { for } y \in\langle x\rangle
$$

as $\pi$ is a Riesz homomorphism. This along with (H2) means that $\left.\pi A_{i}(\cdot)(s)\right|_{\langle x\rangle},(i \in\{1,2\})$ are real-valued solutions of (7) that approximate $\left.\pi F(\cdot)(s)\right|_{\langle x\rangle}$ on $\langle x\rangle$. Hence $\pi A_{1}(x)(s)=$ $\pi A_{2}(x)(s)$. But then $\pi A_{1}(x)=\pi A_{2}(x)$ as $s \in W_{x}$ was chosen arbitrarily, $W_{x}$ is open and dense in $X$, and $\pi A_{1}(x)$ and $\pi A_{2}(x)$ are continuous. Since $\pi$ is injective we infer that $A_{1}(x)=A_{2}(x)$. This completes the proof, as $x \in G$ was arbitrarily fixed.

Remark 8. Theorem 7 remains valid for more involved functional equations, for instance, alternative (conditional) functional equations

$$
\left(\mathscr{E}_{1} \vee \mathscr{E}_{2}\right) F=0
$$

for $\mathscr{E}_{1}, \mathscr{E}_{2}: L^{G} \rightarrow L^{G^{k}}$. By (59) we mean that $F: G \rightarrow L$ satisfies (59) if

$$
\mathscr{E}_{1} F(\bar{x})=0 \quad \text { or } \quad \mathscr{E}_{2} F(\bar{x})=0 \quad \text { for } \bar{x} \in G^{k} .
$$

Given $u_{1}, u_{2} \in L_{+}$, any $F: G \rightarrow L$ is a $\left(u_{1}, u_{2}\right)$-near solution of (59) if

$$
\left|\mathscr{E}_{1} F(\bar{x})\right| \leq u_{1} \quad \text { or } \quad\left|\mathscr{E}_{2} F(\bar{x})\right| \leq u_{2} \quad \text { for } \bar{x} \in G^{k} .
$$

We assume that both operators $\mathscr{E}_{1}$ and $\mathscr{E}_{2}$ satisfy (H2) and (H5). Since, in fact, $\delta$ is now two-place function, we assume that $\Delta\left(u_{1}, u_{2}\right)$ satisfies $\pi \Delta\left(u_{1}, u_{2}\right)(s) \geq \delta\left(\pi u_{1}(s), \pi u_{2}(s)\right)$ for $s \in R\left(\pi \Delta\left(u_{1}, u_{2}\right)\right)$. Moreover, defining open and dense subsets of $X$ we replace any occurrence of $R(\pi u)$ by $R\left(\pi u_{1}\right) \cap$ $R\left(\pi u_{2}\right)$.

Remark 9. Similarly, instead of an alternative in (59) one may consider a conjunction, which is useful to investigate systems of functional equations.

Remark 10. Observe that Theorem 7 remains valid if one considers a slightly more general definition of the URAP. Namely, 
one can allow sequences $\alpha_{n}, \beta_{n}, \gamma_{n}, \rho_{n}$ to be dependent on $x$. Now we assume the convergence of $\alpha_{n}(x), \beta_{n}(x), \rho_{n}(x)$ to 0 and $\gamma_{n}(x)$ to $\gamma(x)$ at each point $x \in G$. Moreover, one can consider different deltas on the right-hand sides of (P1)-(P3).

Remark 11. Let us note that one can replace condition $(\mathrm{P} 3)$ in Definition 2 of the URAP with the following one:

$\left(\mathrm{P}^{\prime}\right)$ the sequence

$$
\xi_{n}:=\sup _{\bar{x} \in G^{k}}\left|\mathscr{E} \mathscr{H}_{n} f(\bar{x})\right|
$$

is convergent to 0 .

Then, accordingly, Step 2 of the proof of Theorem 7, that is, the proof that $A$ defined by (50) satisfies (7), should be replaced by the following reasoning.

Let $\bar{x}=\left(x_{1}, x_{2}, \ldots, x_{k}\right) \in G^{k}$. By (H4) there exists an open and dense subset $w_{\bar{x}}$ of $X$ with (20). Let $W_{\bar{x}}:=w_{\bar{x}} \cap R(\pi u) \cap$ $R(\pi \Delta(u)) \cap R(\pi \mathscr{E} A(\bar{x}))$. By the definition of $A(50)$ and (H5) we have

$$
\lim _{n \rightarrow \infty}{ }^{\Delta} \mathscr{E} \mathscr{H}_{n} F(\bar{x})=\mathscr{E} A(\bar{x})
$$

Let $s \in W_{\bar{x}}$ be fixed. Then,

$$
\lim _{n \rightarrow \infty} \pi \mathscr{E} \mathscr{H}_{n} F(\bar{x})(s)=\pi \mathscr{E} A(\bar{x})(s)
$$

On the other hand, by (H1) and (H2), for any $\bar{y} \epsilon$ $\left\langle x_{1}, x_{2}, \ldots, x_{k}\right\rangle^{k}$, we have

$$
|\mathscr{E} \pi F(\cdot)(s)(\bar{y})| \leq \pi u(s)
$$

which means that $\left.\pi F(\cdot)(s)\right|_{\left\langle x_{1}, x_{2}, \ldots, x_{k}\right\rangle}$ is a real-valued $\pi u(s)$ solution of (7) on $\left\langle x_{1}, x_{2}, \ldots, x_{k}\right\rangle$. Since (7) has the URAP, then $\left(\mathrm{P}^{\prime}\right)$, (H2), and (H3) yield

$$
\lim _{n \rightarrow \infty} \pi \mathscr{E} \mathscr{H}_{n} F(\bar{x})(s)=\lim _{n \rightarrow \infty} \mathscr{E} \mathscr{H}_{n} \pi F(\cdot)(s)(\bar{x})=0 .
$$

Taking into account (64), we have

$$
\pi \mathscr{E} A(\bar{x})(s)=0 .
$$

Since the last equality is valid for any $s$ from the open and dense subset $W_{\bar{x}}$ of $X$ and all the functions in the above inequality are continuous, we obtain

$$
\pi \mathscr{E} A(\bar{x})=0
$$

Consequently, we infer that

$$
\mathscr{E} A(\bar{x})=0
$$

as $\pi$ is a Riesz isomorphism. This, due to the fact that $\bar{x} \in G^{k}$ was chosen arbitrarily, completes the proof that $A$ satisfies (7).

\section{Approximate Solutions of an Alternative Cauchy Equation}

In this section we deal with approximate solutions of an alternative Cauchy functional equation

$F(x+y)+F(x)+F(y) \neq 0 \Longrightarrow F(x+y)=F(x)+F(y)$.

This equation belongs to the class of conditional Cauchy equations with the condition dependent on the unknown function. The general solution of (70) is described in [15, Theorem 8]. Stability of this equation, in the class of functions mapping an Abelian semigroup into a Banach space, has been investigated in [16] and in a more general setting in [17]. For the readers convenience we quote the main result of [16] as it will be used in the sequel.

Theorem 12 (cf. [16, Theorem 1]). Let $(G,+)$ be an Abelian semigroup and let $(E,\|\cdot\|)$ be a Banach space. If, for some $\varepsilon_{1}, \varepsilon_{2} \geq 0$ and all $x, y \in G$, a function $f: G \rightarrow$ Esatisfies

$$
\begin{aligned}
\| f(x+y) & +f(x)+f(y) \| \\
& >\varepsilon_{1} \Longrightarrow\|f(x+y)-f(x)-f(y)\| \leq \varepsilon_{2},
\end{aligned}
$$

then there exists a unique additive function $a: G \rightarrow E$ such that

$$
\|f(x)-a(x)\| \leq \max \left\{\varepsilon_{1}, \varepsilon_{2}\right\}
$$

for all $x \in G$.

The natural question arises if a similar result holds true in ordered spaces. One can rewrite all the sentences of Theorem 12 for functions mapping an Abelian semigroup $G$ into a Riesz space $L$, replacing the norm by the absolute value in $L$. The main goal of this section is to apply Theorem 7 with the purpose to give an affirmative answer to this question.

We will use one of the most general spectral representation theorems, namely, the Johnson-Kist Spectral Representation Theorem which we quote here.

Theorem 13 (Johnson-Kist representation theorem) (cf. [8, Theorem 44.4]). Let L be an Archimedean Riesz space. There exists a Riesz space $\widehat{L}$ of extended real continuous functions and a Riesz isomorphism of L onto $\widehat{L}$.

The main theorem of this section reads as follows.

Theorem 14. Let $(G,+)$ be an Abelian semigroup and let $L$ be an Archimedean Riesz space. Assume that, for given $u_{1}, u_{2} \in$ $L_{+}, L$ is $\sup \left\{u_{1}, u_{2}\right\}$-uniformly complete. If, for every $x, y \in G$, a function $F: G \rightarrow$ L satisfies

$$
\begin{gathered}
|F(x+y)+F(x)+F(y)| \leq u_{1} \quad \text { or } \\
|F(x+y)-F(x)-F(y)| \leq u_{2},
\end{gathered}
$$

then there exists a unique additive function $A: G \rightarrow L$ such that

$$
|F(x)-A(x)| \leq \sup \left\{u_{1}, u_{2}\right\} \quad \text { for } x \in G \text {. }
$$


Proof. By the Johnson-Kist Spectral Representation Theorem there exist a topological space $X$ and a Riesz isomorphism $\pi: L \rightarrow \widehat{L} \subset C^{\infty}(X)$; hence $(\mathrm{H} 1)$ is satisfied. (H2' ${ }^{\prime}$ ) and (H5) hold because (70) has the form (59) with $\mathscr{E}_{1} F(x, y):=$ $F(x+y)+F(x)+F(y)$ and $\mathscr{E}_{2} F(x, y):=F(x+y)-F(x)-F(y)$, for $x, y \in G$. If $F$ satisfies (73) then

$$
|F(x+y)| \leq|F(x)+F(y)|+\sup \left\{u_{1}, u_{2}\right\} \quad \text { for } x, y \in G
$$

and, therefore, (L2) holds with $B(v):=|v|, C(v, w):=\mid v+$ $w \mid+\sup \left\{u_{1}, u_{2}\right\}$ for $v, w \in L$ and $R_{C}:=R\left(\pi \sup \left\{u_{1}, u_{2}\right\}\right)$. Thus, by Lemma 6, we have (H4). It is clear that $\mathscr{H}_{n}$ : $L^{G} \rightarrow L^{G}$ given by $\mathscr{H}_{n} F(x):=(1 / n) F(n x)$, for $x \in G$, $n \in \mathbb{N}$, satisfies (H3). Moreover, by Theorem 12 applied for $E=\mathbb{R}$ and Lemma 3, one can easily verify that (59) possesses URAP with $\alpha_{n}=\beta_{n}=1 / n, \gamma_{n}=1+1 / n, \rho_{n}=3 / n$ for $n \in \mathbb{N}$ and $\delta\left(\varepsilon_{1}, \varepsilon_{2}\right)=\sup \left\{\varepsilon_{1}, \varepsilon_{2}\right\}$. Putting $\Delta\left(u_{1}, u_{2}\right)=$ $\sup \left\{u_{1}, u_{2}\right\}$ we have $\pi \Delta\left(u_{1}, u_{2}\right)=\sup \left\{\pi u_{1}, \pi u_{2}\right\}$ which means that $\pi \Delta\left(u_{1}, u_{2}\right)(s)=\sup \left\{\pi u_{1}(s), \pi u_{2}(s)\right\}=\delta\left(\pi u_{1}(s), \pi u_{2}(s)\right)$ for $s \in R\left(\pi \Delta\left(u_{1}, u_{2}\right)\right)$.

Now all the assertions of Theorem 14 follow directly from Theorem 7 .

It is easy to observe that the constant of approximation in Theorem 14 is the best possible one.

In view of Theorem 12 and the meaning of the approximate solution of an alternative functional equation (70), that is condition (71) one may expect the following implication:

$$
\begin{aligned}
& |F(x+y)+F(x)+F(y)| \\
& \quad>u_{1} \Longrightarrow|F(x+y)-F(x)-F(y)| \leq u_{2},
\end{aligned}
$$

for $x, y \in G$, in Theorem 14 instead of condition (73), with the common meaning of $a<b$ as $a \leq b$ and $a \neq b$. Of course, if the order in a Riesz space $L$ is linear then conditions (73) and (76) coincide. However, as it will be shown in the example below, in general, assumption (73) cannot be replaced by (76).

Example 15. Let $L$ be the Archimedean Riesz space of all real functions of real variable with the pointwise order and let $u_{1} \in$ $L$ be given by

$$
u_{1}(s):=\left\{\begin{array}{ll}
1, & \text { if } s=0 \\
0, & \text { if } s \neq 0
\end{array} \quad \text { for } s \in \mathbb{R} .\right.
$$

Then $L$ is $u_{1}$-uniformly complete. We define $F: \mathbb{R} \rightarrow L$ by

$$
F(x)(s):=\left\{\begin{array}{ll}
x, & \text { if } s=x \\
0, & \text { if } s \neq x
\end{array} \quad \text { for } x, s \in \mathbb{R} .\right.
$$

Then $F$ is not additive and satisfies (76) with $u_{1}$ defined above and $u_{2} \equiv 0$. On the other hand, $F$ cannot be approximated by any additive function. Suppose, for contradiction, that there exists an additive mapping $A: \mathbb{R} \rightarrow L$ satisfying (74). Let us fix $x \in \mathbb{R}$. For $s \neq 0$ inequality (74) results with $F(x)(s)=$ $A(x)(s)$ according to the definition of $u_{1}$. Directly from the definition of $F$ we have $F(x)(0)=0$. Then by (74) and the additivity of $A$ we obtain $A(x)(0)=0$. Eventually, we infer that $F$ and $A$ coincide and, therefore, $F$ is additive. We have obtained a contradiction.
Let us point out that the assumption that the Riesz space $L$ is Archimedean is necessary in order to have the uniqueness of an existing additive function $A$ in Theorem 14 , which can be observed in the following simple example.

Example 16. Let us consider the lexicographically ordered plane $L=\mathbb{R}^{2} . L$ is then $(1,0)$-uniformly complete Riesz space. Moreover function $F: \mathbb{R} \rightarrow \mathbb{R}^{2}$ given by $F(x)=(x, 0)$ satisfies inequality (73) with $u_{1}=(1,0)$ and $u_{2}=(0,0)$. On the other hand inequality (74) holds true with any additive mapping $A_{\alpha}: \mathbb{R} \rightarrow \mathbb{R}^{2},(\alpha \in \mathbb{R})$, of the form $A_{\alpha}(x)=$ $(x, \alpha x)$.

\section{Approximate Solutions of the Cauchy Equation with Squares}

Equation (70) has stemmed from

$$
f(x+y)^{2}=(f(x)+f(y))^{2},
$$

with a real function $f$, and next has been investigated in the form

$$
|f(x+y)|=|f(x)+f(y)|
$$

which admits further generalisations from the real case to more general structures. Affirmative results concerning stability of (80) are contained in [18] for real-valued functions and, for the class of functions taking values in Riesz spaces, in [5]. There are also known results concerning the stability of the generalized equation (80) for functions acting into a normed space, called Fischer-Muszély functional equation:

$$
\|f(x+y)\|=\|f(x)+f(y)\| \quad \text { for } x, y \in G .
$$

It occurs that, despite the fact that on the assumption that the norm is strictly convex (81) is equivalent to the Cauchy functional equation (cf. [19]), even if we consider $\mathbb{R}^{2}$ with the Euclidean norm as a target space of $f,(81)$ fails to be stable in the Hyers-Ulam sense (cf. [18]). However, if we consider the stability of (81) in the class of surjective functions, then the answer is positive (cf. [20]).

Finally, concerning (79) in the class of complex functions we have the following stability result.

Theorem 17 (cf. [16, Theorem 2]). Let $(G,+)$ be an Abelian semigroup. If for a given $\varepsilon \geq 0$ a function $f: G \rightarrow \mathbb{C}$ satisfies

$$
\left|f(x+y)^{2}-(f(x)+f(y))^{2}\right| \leq \varepsilon \quad \text { for } x, y \in G,
$$

then there exists a unique additive function $a: G \rightarrow \mathbb{C}$ such that

$$
|f(x)-a(x)| \leq \sqrt{\varepsilon} \text { for } x \in G
$$

Remark 18. In fact, for complex functions, (79) occurs to be superstable in the sense of Baker which was proved in [21] and, with the use of Theorem 5, in [22]. 
The main aim of this section is to apply Theorem 7 in order to prove that in the class of functions taking values in an $f$-algebra equation (79) is stable; however it is not superstable. Our main stability result reads as follows.

Theorem 19. Let $(G,+)$ be an Abelian semigroup and let $L$ be an Archimedean $f$-algebra with a multiplicative identity $e \in$ $L_{+}$. Assume that $L$ is $u$-uniformly complete for given $u \in L_{+}$. If a function $F: G \rightarrow L$ satisfies

$$
\left|F(x+y)^{2}-(F(x)+F(y))^{2}\right| \leq u^{2} \quad \text { for } x, y \in G,
$$

then there exists a unique additive function $A: G \rightarrow L$ such that

$$
|F(x)-A(x)| \leq u \quad \text { for } x \in G .
$$

In the proof of Theorem 19 we are going to use Proposition 2 from [3], which is quoted below. This proposition is due to the Ogasawara-Maeda Spectral Representation Theorem for Archimedean Riesz spaces with a weak unit (cf. [8, Theorem 50.1]). The multiplicative identity is a weak order unit (cf. [9, Proposition 353P]) and the topological spacethe domain of the Ogasawara-Maeda representativesappears to be extremally disconnected; hence, according to Proposition $1, C^{\infty}(X)$ is an $f$-algebra.

Proposition 20 (cf. [3, Proposition 2]). Let $L$ be an Archimedean $f$-algebra with a multiplicative identity e $\in L_{+}$. Then there exist a topological space $X$ and an $f$-subalgebra $\widehat{L}$ of the $f$-algebra $C^{\infty}(X)$ and an $f$-algebra isomorphism of L onto $\widehat{L}$.

Proof of Theorem 19. By Proposition 20 there exist a topological space $X$ and an $f$-algebra isomorphism $\pi: L \rightarrow \widehat{L} \subset$ $C^{\infty}(X)$; hence (H1) is satisfied. Equation (79) has the form (7) with $\mathscr{E} F(x, y):=F(x+y)^{2}-(F(x)+F(y))^{2}$, for $x, y \in G$; thus (H2) and (H5) hold. By (84) we have

$$
\left|F(x+y)^{2}\right| \leq\left|(F(x)+F(y))^{2}\right|+u^{2} \quad \text { for } x, y \in G
$$

and, therefore, (L2) holds with $B(v)=\left|v^{2}\right|, C(v, w):=\mid(v+$ $w)^{2} \mid+u^{2}$ for $v, w \in L$ and $R_{C}:=R\left(\pi u^{2}\right)$. Thus, by Lemma 6 we have (H4). It is evident that $\mathscr{H}_{n}: L^{G} \rightarrow L^{G}$ given by $\mathscr{H}_{n} F(x):=(1 / n) F(n x)$ for $x \in G, n \in \mathbb{N}$ satisfies (H3). Moreover, by Theorem 17 and Lemma 3 (79) possesses the URAP with $\alpha_{n}=\beta_{n}=1 / n, \gamma_{n}=1+1 / n$, and $\rho_{n}=3 / n$ for $n \in \mathbb{N}$ and $\delta\left(\varepsilon^{2}\right)=\varepsilon$. For $\Delta\left(u^{2}\right)=u$ we have $\pi \Delta\left(u^{2}\right)=\pi u$ and consequently $\pi \Delta\left(u^{2}\right)(s)=\pi u(s)=\delta\left((\pi u(s))^{2}\right)$ for $s \in$ $R\left(\pi \Delta\left(u^{2}\right)\right)$.

Having applied Theorem 7 we finish the proof.

Theorem 19 states that in $f$-algebras the Cauchy equation with squares (79) is stable in the Hyers-Ulam sense. According to Remark 18 one can ask if (79) is superstable in the sense of Baker. But it appears that this is not the case.

Example 21. Let $B[-1,1]$ be the Archimedean $f$-algebra of all bounded real functions on the interval $[-1,1]$ with a multiplicative identity $e \equiv 1$, the pointwise order, pointwise addition, and multiplication. Let $u \in B[-1,1]$ be given by

$$
u(s):= \begin{cases}\sqrt{3}|s|, & \text { if } s \in[-1,0] \\ 0, & \text { if } s \in(0,1] .\end{cases}
$$

Then $B[-1,1]$ is $u$-uniformly complete and $F: \mathbb{R} \rightarrow B[-1,1]$ given by

$$
F(x)(s):=\left\{\begin{array}{ll}
s, & \text { if } s \in[-1,0] \\
s x, & \text { if } s \in(0,1]
\end{array} \text { for } x \in \mathbb{R}\right.
$$

is, clearly, neither bounded nor additive. Moreover, one can easily check that $F$ and $u$ satisfy (84).

\section{Approximate Solutions of the Quadratic Functional Equation}

In this section we deal with approximate solutions of the quadratic functional equation

$$
F(x+y)+F(x-y)=2 F(x)+2 F(y) .
$$

Stability of this equation, in the class of functions mapping an Abelian group into a Banach space, has been investigated in [23] (cf., e.g., [24-26]).

Theorem 22 (cf. [23]). Let $(G,+)$ be an Abelian group and let $(E,\|\cdot\|)$ be a Banach space. If a function $f: G \rightarrow E$ satisfies the inequality

$$
\|f(x+y)+f(x-y)-2 f(x)-2 f(y)\|<\varepsilon
$$

for some $\varepsilon \geq 0$ and all $x, y \in G$ then there exists a unique quadratic function $q: G \rightarrow$ E such that

$$
\|f(x)-q(x)\| \leq \frac{1}{2} \varepsilon
$$

for all $x \in G$.

The main aim of this section is to show that a similar result holds true in the class of Riesz space-valued mappings.

Theorem 23. Let $(G,+)$ be an Abelian group and let $L$ be an Archimedean u-uniformly complete Riesz space, with some $u \in$ $L_{+}$. If, for every $x, y \in G$, a function $F: G \rightarrow L$ satisfies

$$
|F(x+y)+F(x-y)-2 F(x)-2 F(y)| \leq u,
$$

then there exists a unique quadratic function $Q: G \rightarrow L$ such that

$$
|F(x)-Q(x)| \leq \frac{1}{2} u \quad \text { for } x \in G .
$$

Proof. By the Johnson-Kist Spectral Representation Theorem there exist a topological space $X$ and a Riesz isomorphism $\pi: L \rightarrow \widehat{L} \subset C^{\infty}(X)$; hence (H1) is satisfied. Equation (89) 
has the form (7) with $\mathscr{E} F(x, y):=F(x+y)+F(x-y)-2 F(x)-$ $2 F(y)$; hence (H2) and (H5) hold. If $F$ satisfies (92) then

$$
\begin{array}{r}
|F(x+y)| \leq|F(x-y)-2 F(x)-2 F(y)|+u \\
\text { for } x, y \in G
\end{array}
$$

and therefore (L3) holds with $B(v):=|v|, D(t, v, w):=\mid t-$ $2 v-2 w \mid+u$ for $t, v, w \in L$ and $R_{D}:=R(\pi u)$. Consequently Lemma 6 guaranties (H4). It is clear that $\mathscr{H}_{n}: L^{G} \rightarrow L^{G}$ given by $\mathscr{H}_{n} F(x):=F\left(2^{n} x\right) / 4^{n}$ for $x \in G, n \in \mathbb{N}$ satisfies (H3). Moreover, by Theorem 22 applied for $E=\mathbb{R}$ one can easily verify that (89) possesses URAP with $\left(\mathscr{H}_{n} F(x)\right)_{n \in \mathbb{N}}$ defined above and $\alpha_{n}=\beta_{n}=1 / 4^{n}, \gamma_{n}=1+1 / 4^{n}, \delta_{n}=3 / 4^{n}$ for $n \in \mathbb{N}, \delta(\varepsilon)=\varepsilon / 2$. Putting $\Delta(u)=u / 2$ we have $\pi \Delta(u)(s)=$ $(\pi u(s)) / 2=\delta(\pi u(s))$ for $s \in R(\pi \Delta(u))$.

Applying Theorem 7 we finish the proof.

\section{Conflict of Interests}

The author declares that there is no conflict of interests regarding the publication of this paper.

\section{Acknowledgment}

The author wishes to express gratitude to the anonymous referee for several valuable suggestions.

\section{References}

[1] B. Batko, "Stability of the exponential functional equation in Riesz algebras," Abstract and Applied Analysis, vol. 2014, Article ID 848540, 4 pages, 2014.

[2] B. Batko, "The stability of Dhombres' equation in Riesz spaces," Journal of Mathematical Analysis and Applications, vol. 406, no. 1, pp. 261-265, 2013.

[3] B. Batko, "Spectral representation theory and stability of the multiplicative Dhombres functional equation in f-algebras," Aequationes Mathematicae, 2014.

[4] B. Batko and J. Brzdęk, "A fixed point theorem and the Hyers-Ulam stability in Riesz spaces," Advances in Difference Equations, vol. 2013, article 138, 2013.

[5] B. Batko and J. Tabor, "Stability of the generalized alternative Cauchy equation," Abhandlungen aus dem Mathematischen Seminar der Universität Hamburg, vol. 69, pp. 67-73, 1999.

[6] F. Polat, "Approximate Riesz algebra-valued derivations," Abstract and Applied Analysis, vol. 2012, Article ID 240258, 5 pages, 2012.

[7] F. Polat, "Some generalizations of Ulam-Hyers stability functional equations to Riesz algebras," Abstract and Applied Analysis, vol. 2012, Article ID 653508, 9 pages, 2012.

[8] W. A. J. Luxemburg and A. C. Zaanen, Riesz Spaces, NorthHolland, Amsterdam, The Netherlands, 1971.

[9] D. H. Fremlin, Measure Theory: Measure Algebras, vol. 3, Torres Fremlin, Colchester, UK, 2002.

[10] S. M. Ulam, A Collection of Mathematical Problems, Interscience Publishers, New York, NY, USA, 1960.

[11] D. H. Hyers, "On the stability of the linear functional equation," Proceedings of the National Academy of Sciences of the United States of America, vol. 27, pp. 222-224, 1941.
[12] G. Pólya and G. Szegö, Aufgaben Und Lehrsätze Aus Der Analysis I, Julius Springer, Berlin, Germany, 1925.

[13] Z. Moszner, "Sur les définitions différentes de la stabilité des équations fonctionnelles," Aequationes Mathematicae, vol. 68, no. 3, pp. 260-274, 2004.

[14] N. Brillouët-Belluot, J. Brzdęk, and K. Ciepliński, "On some recent developments in Ulam's type stability," Abstract and Applied Analysis, vol. 2012, Article ID 716936, 41 pages, 2012.

[15] J. G. Dhombres and R. Ger, "Conditional Cauchy equations," Glasnik Matematički, vol. 13, no. 1, pp. 39-62, 1978.

[16] B. Batko, "On the stability of an alternative functional equation," Mathematical Inequalities \& Applications, vol. 8, no. 4, pp. 685691, 2005.

[17] B. Batko, "Stability of an alternative functional equation," Journal of Mathematical Analysis and Applications, vol. 339, no. 1, pp. 303-311, 2008.

[18] B. Batko and J. Tabor, "Stability of an alternative Cauchy equation on a restricted domain," Aequationes Mathematicae, vol. 57, no. 2-3, pp. 221-232, 1999.

[19] R. Ger, "On a characterization of strictly convex spaces," Atti della Accademia delle Scienze di Torino, vol. 127, no. 3-4, pp. 131138, 1993.

[20] J. Tabor, "Stability of the Fischer-Muszély functional equation," Publicationes Mathematicae Debrecen, vol. 62, no. 1-2, pp. 205211, 2003.

[21] P. W. Cholewa, "Stability as a property dependent upon an equation, not on a function in: Report of Meeting, The 34th International Symposium on Functional Equations," Aequationes Mathematicae, vol. 53, no. 1-2, pp. 162-205, 1997.

[22] J. Schwaiger, "Remark 13 in: Report of Meeting, The 41st International Symposium on Functional Equations," Aequationes Mathematicae, vol. 67, p. 309, 2004.

[23] P. W. Cholewa, "Remarks on the stability of functional equations," Aequationes Mathematicae, vol. 27, no. 1-2, pp. 76-86, 1984.

[24] F. Skof, "Proprieta locali e approssimazione di operatori," Rendiconti del Seminario Matematico e Fisico di Milano, vol. 53, pp. 113-129, 1983.

[25] S. Czerwik, "On the stability of the quadratic mapping in normed spaces," Abhandlungen aus dem Mathematischen Seminar der Universität Hamburg, vol. 62, pp. 59-64, 1992.

[26] F. Skof and S. Terracini, "Sulla stabilita dell'equazione funzionale quadratica su un dominio ristretto," Atti della Accademia delle Scienze di Torino. Classe di Scienze Fisiche Matematiche e Naturali, vol. 121, pp. 153-167, 1987. 


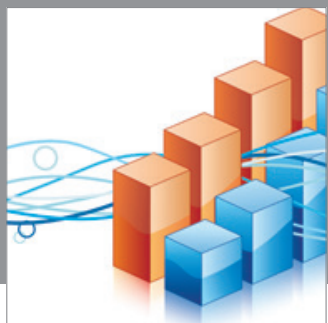

Advances in

Operations Research

mansans

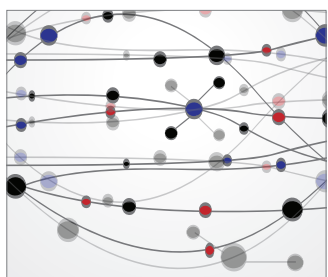

The Scientific World Journal
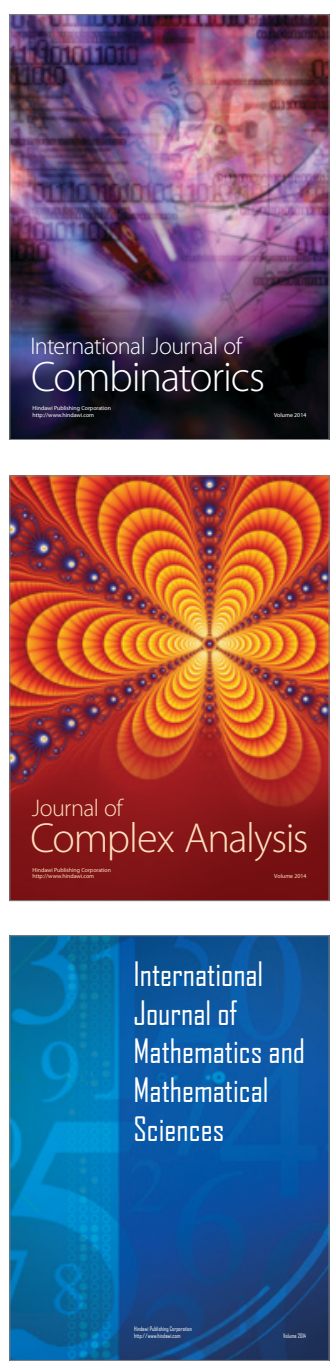
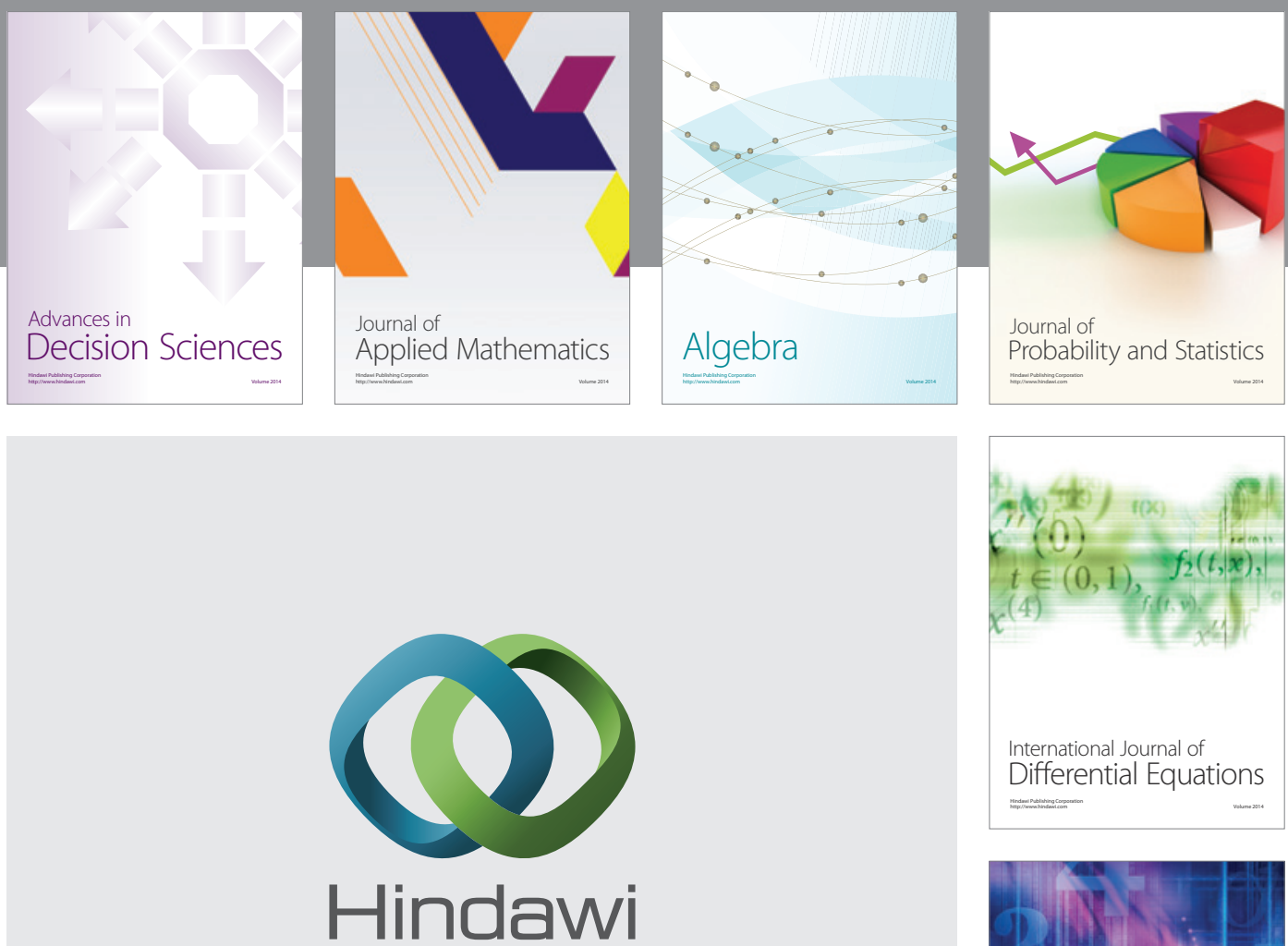

Submit your manuscripts at http://www.hindawi.com
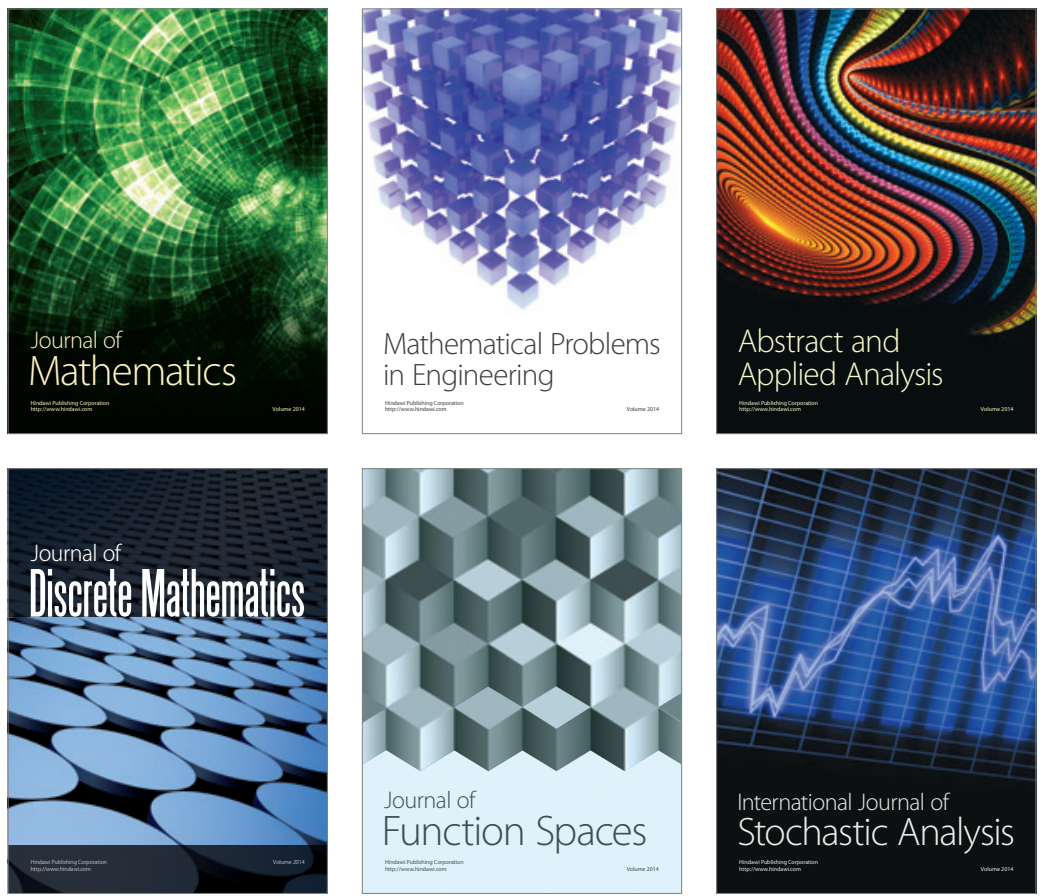

Journal of

Function Spaces

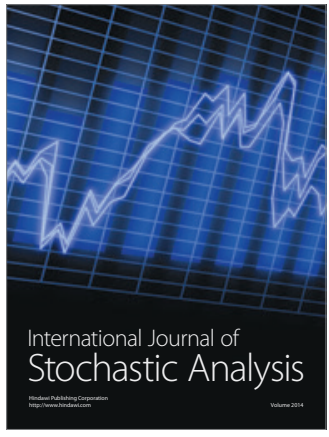

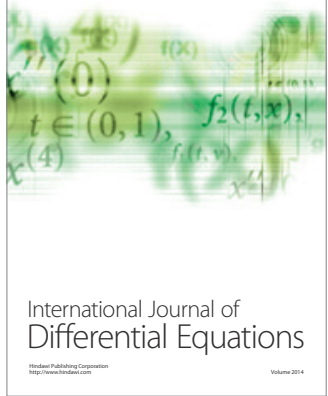
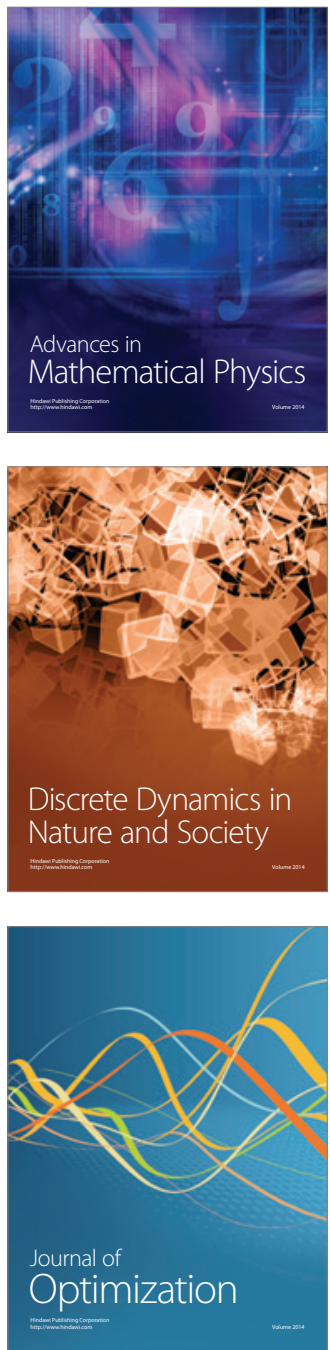